\title{
Impacts of COVID-19 Pandemic for Software Development in Nordic Companies - Agility Helps to Respond
}

\author{
Petri Kettunen $^{1(\otimes)}\left(\mathbb{D}\right.$, Tomas Gustavsson ${ }^{2}$ (D), Maarit Laanti ${ }^{3}$, Andreas Tjernsten $^{4}$, \\ Tommi Mikkonen $^{1}$ (D), and Tomi Männistö ${ }^{1}$ (D) \\ ${ }^{1}$ University of Helsinki, Helsinki, Finland \\ \{petri.kettunen, tommi.mikkonen, tomi.mannisto\}@helsinki.fi \\ 2 Karlstad University, Karlstad, Sweden \\ tomas.gustavsson@kau.se \\ 3 Nitor Delta, Helsinki, Finland \\ maarit.laanti@nitor.com \\ ${ }^{4}$ Nitor Agile AB, Stockholm, Sweden \\ andreas.tjernsten@initor.com
}

\begin{abstract}
In 2020, the global COVID-19 pandemic affected almost every company in some way also in the Nordic countries. Depending on the different industry sectors of the companies, the impacts have varied from minor risks to severe disruptions but also even booming businesses. In all, agility and resilience have been required to continue and even to survive. In 2018, we started conducting large-scale agile surveys in Finland and Sweden. For the 2020 survey round, we included questions about the current pandemic situation impacts and how agility has helped to respond. The respondents represented software professionals from different industries, not limited to information and communication technology (ICT) companies. The results indicate that although the perceived impacts have mostly been negative (53\%), it is not all so. One-third (33\%) reported positive impacts such as increased business and better well-being. The majority $(55 \%)$ of the responses indicated that agility has helped to respond to the pandemic situation. Remarkably, 59\% reported that their companies have improved agility during the past year. Improved agility appears to be positively related to the ability to respond to the pandemic. We did not discover significant differences between the Finnish and Swedish respondent cohorts.
\end{abstract}

Keywords: Agility $\cdot$ COVID-19 $\cdot$ Digitalization $\cdot$ Agile $\cdot$ Scaled agile

\section{Introduction}

In 2020, the COVID-19 disease spread across the world and was declared a "Public Health Emergency of International Concern" by the World Health Organization (WHO). Governments enforced regulations and proposed recommendations to prevent further spread of the virus. Technology companies all over the world locked down their offices and made their employees work from home. Many businesses were suddenly disrupted. 
We investigated the pandemic phenomenon based on a recent survey. The survey was conducted during October-November 2020. The overall purpose of the survey was to understand the state of agility in the Nordic countries. This study aimed at answering how the current situation of the pandemic has impacted companies and how well agility helps companies to respond to the situation.

\section{Background}

Current studies reflect impacts on software professionals during the first months of the COVID-19 pandemic. Working from home at a scale never seen before under these new conditions is very much different from normal working from home. In one survey among software professionals, the results show evidence of declining productivity and well-being [1]. They also found an indication of a disproportionately negative effect on women, parents, and people with disabilities. Another investigation found that working from home during the pandemic has different impacts on the productivity of software professionals, depending on which metrics to use [2]. The impacts also differed based on project type, size, and age of employees. A study of GitHub activities presents that patterns of activity of software professionals might have implications for burnout [3]. In addition, the study suggests that the cadence of work has changed since working days have become longer by up to an hour a day, on both weekdays and weekends.

Overall, the pandemic has affected different industries and companies in different countries in various ways, even within the core ICT industry, and different companies have devised various ways to respond and adapt to the impacts (e.g., [4, 5]). The pandemic year 2020 affected significantly and suddenly the work-life in Finland and Sweden too, due to extensive telecommuting recommendations. Full-time remote working has not been typical in either Finnish or Swedish companies in general.

Because agile companies and software organizations are by definition capable of coping with and adapting to changes in their environments and operations, we expected that they were able to respond to the impacts of the pandemic in fitting ways $[6,7]$. Moreover, some companies may even have been able to deal with the disruptions as new opportunities causing positive impacts. However, agile practices are based on dedicated teams that interact closely with each other and the customer. Teams collaborating in close proximity and communicating in daily face-to-face meetings are typical characteristic of agile. But since remote working does not allow agile practices to be performed in the previous way, practices and tools needed to be replaced. This significantly changed the agile way of working [5].

\section{Research Design and Method}

The research effort started in Finland in 2018 from an industrial stance. The initial idea of this survey research endeavor was to investigate the current state of agile software development in Finnish companies. We were interested in refreshing how companies currently use agile methods and how agile they really are. In 2018 and 2019, we conducted two survey rounds, the first year in Finland and the second in Sweden. We have 
reported those results in our prior publications [8]. The results in this paper do not include answers from those earlier rounds.

Overall, the purpose of our survey research was to examine the current state of agile development and enterprise agility. Different companies may approach agile development and agility in different ways. Hence, we were interested in examining how agile companies really are nowadays and how they currently practice agile software development. We are interested in measuring how widely agile methods and practices are currently applied in industrial practice and how that is evolving. Moreover, we want to go beyond team levels to large-scale agile and enterprise agility. We seek to understand why different companies want to change - even transform - with agile means and how beneficial and successful their particular changes have been. In all, we targeted to investigate not just ICT companies but industries in general. The target population was intentionally not limited to software companies since we were also interested in non-software companies (i.e., companies in other industries than IT) currently facing digitalization and becoming more software-intensive. We were also interested in the future. We aimed to investigate not only the current whereabouts but also the future intentions of the companies.

The research method was a descriptive survey. The questions and the predefined answer choices were compiled by referring to selected prior surveys and by deriving from own experiences and research. Most of the questions were closed-type with an open free-text choice. Certain questions depended on their preceding questions. The draft questionnaire was first piloted both in our industrial and academic organizations.

In 2020, we revised the questionnaire based on the experiences of the 2018-2019 survey. We added some new questions, removed some of the previous ones, and refined some. In particular, we introduced a section of the current situation of the pandemic, which emerged during the questionnaire revision period. The revised version of the questionnaire comprised in total 48 question items with Finnish, Swedish, and English language variants (translated by the native Finnish and Swedish speaking authors of this paper). Again, certain of them depended on the selector question answers. The content questions (except the selector ones) were non-mandatory and had "I don't know" and N/A options. A pilot round was conducted.

For data collection, the survey was implemented with a web-based online questionnaire tool. We considered several potential distribution channels in order to reach a wide, representative sample population. However, due to pragmatic constraints, we decided to use convenience sampling. The questionnaire was distributed through a Finnish consulting company newsletter to people (300) mainly in Finland and Sweden who are interested in the company's offering of software consultancy, training, and agile transformation services, as well as shared through several social media channels, especially by posting links to the survey in agile user-groups on LinkedIn and Facebook. In addition, we advocated it to certain software research interest groups. The survey was open for seven weeks during October and November 2020. We received 137 responses.

\section{Results}

To address the research objectives described above, we analyzed the respondents' answers to the following survey questions: 
- Q0: How do you see that Your company's overall agility has changed during the past year?

- Q1: How much and in what way does the current situation of global pandemic impact Your company?

- Q2: How has the current situation of global pandemic impacted Your company?

- Q3: How well does agility help Your company to respond to the situation?

Figure 1 illustrates the basic descriptive statistics of the respondents and organizations. 54\% of the respondents were located in Finland, $43 \%$ in Sweden. Software development and software process development were the most frequently reported roles. $66 \%$ of the respondents are in large or very large companies. Notably, two thirds $(67 \%)$ of the respondents are in other sectors than core ICT businesses (computer programming, consultancy, and related activities). Agile methods are widely used.

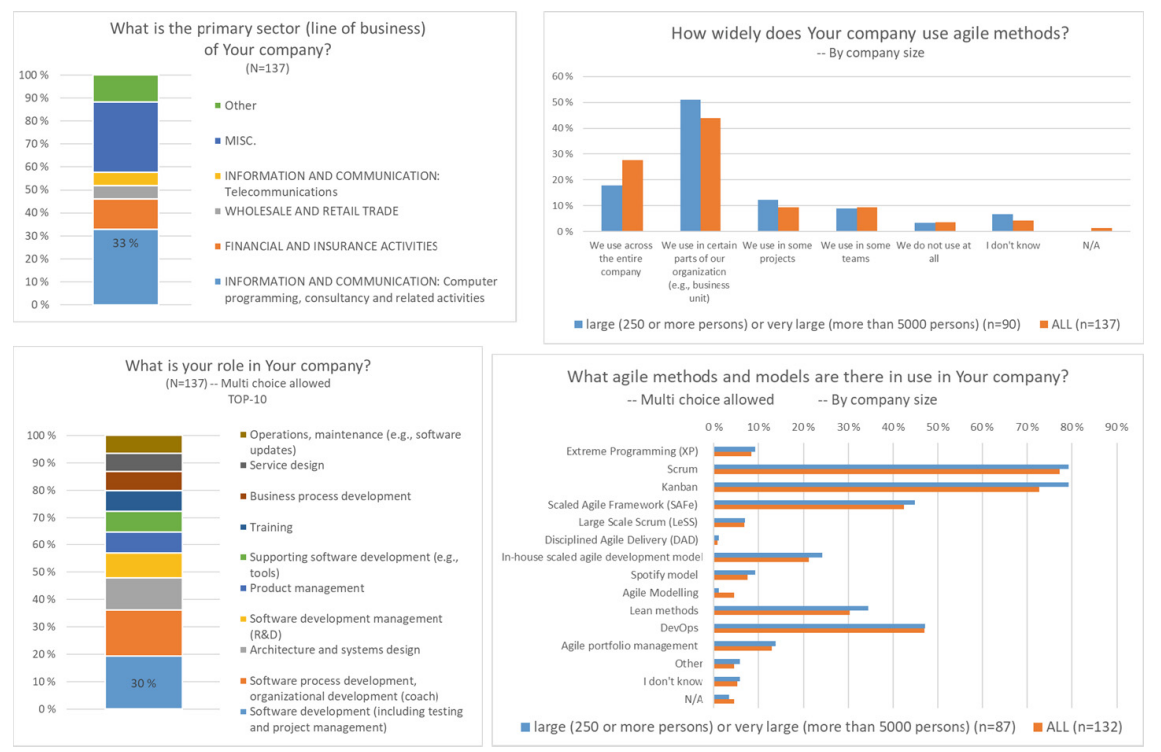

Fig. 1. Demographical information of the respondents and their organizations.

\subsection{How Companies' Overall Agility has Changed (Q0)}

The results about how the respondents perceived that the company's overall agility has changed during the past year (i.e., at the time of the survey in October-November 2020) reported were as follows $(\mathrm{n}=132$ (87 of large/very large companies)): $59 \%$ (69\%) improved (42\% (46\%) a little, 17\% (23\%) significantly), $8 \%(6 \%)$ declined (a little or significantly), and $23 \%$ (20\%) remained the same. Notably, the majority reported improved agility. The distribution in large/very large companies was similar to the all. There appeared to be no significant differences between Finland and Sweden. 


\subsection{How Much and in What Way the Current Global Pandemic Impacts (Q1)}

The purpose of this question is to probe, how positively/negatively the pandemic was perceived to impact (answer scale [extremely neg., extremely pos.]). Aggregating the results, about a half $(53 \%)$ of the respondents $(n=135$ ( 89 of large/very large companies)) reported negative impacts while one third (33\% (34\%)) reported positive impacts (3\% (2\%) even extremely). The distribution in large/very large companies was similar to the all. In all the distribution was slightly more on the positive side in Finland than in Sweden (Finland 51\% negatively, 36\% positively; Sweden 58\% negatively, 29\% positively).

The ICT sector reported being impacted more negatively (50\% negatively, 39\% positively) than the other main sectors of the respondents. Interestingly, the wholesale and retail trade sector appear to have experienced more positive impacts (38\% negatively, $63 \%$ positively), while the financial and insurance sector reported equally positive and negative impacts (39\% negatively, 39\% positively).

\subsection{How the Current Situation of Global Pandemic has Impacted (Q2)}

Following the Q1 (Sect. 4.2), Table 1 displays how the pandemic situation affected companies in practice. The responses to this question are open comments answered by 80 of the respondents. The remaining respondents $(\mathrm{Q} 1 \mathrm{n}=135)$ did not answer this question. The replies (Finnish and Swedish translations into English) were coded in twelve descriptive themes, and the themes were further grouped as positive (POS), negative (NEG), and miscellaneous (MISC) ones, based on the impact on the company. The percentages displayed in Table 1 are based on the number of answers coded for each theme and should be seen as an indication of differences in reported impacts.

Notably, there are both positive and negative impacts reported on business and wellbeing. A declining business was the most often reported impact both in Finland and in Sweden, emphasized in Finland.

An increase in remote working was also reported noticeably more often in Finland. One might expect answers relating to remote work (Remote working increased), and this survey showed this theme with the second highest number of answers. The reported negative impact of Cooperation difficulties was often perceived as an effect of being forced to work from home, while a positive impact was that the situation has in several organizations led to Improved digitalization.

Several answers categorized in the theme Declining business show different amounts of lost customer projects and sales. Some answers show that one impact is an Impeded development situation, implying that improvement activities have been halted. Also, Layoffs and reduction of work hours were reported. However, the survey shows several positive changes for some organizations. These include an Increased business situation while some organizations perceived No big change, that business could continue as usual.

Some responses showed a notion of Declining well-being regarding problems of lowered job satisfaction and well-being. It is clear that the experience differs, however, since some respondents perceived Increased well-being. 
Table 1. (Q2) How has the current situation of global pandemic impacted Your company?

\begin{tabular}{l|l|l|l|l}
\hline \multirow{2}{*}{ Themes } & \multirow{2}{*}{ Type } & \multicolumn{2}{l}{$\%$ of responses } & \multicolumn{2}{l}{} \\
\cline { 3 - 6 } & & ALL $(\mathrm{n}=80)$ & Finland $(\mathrm{n}=39)$ & Sweden $(\mathrm{n}=41)$ \\
\hline Declining business & NEG & $18 \%$ & $23 \%$ & $12 \%$ \\
\hline Cooperation difficulties & NEG & $8 \%$ & $5 \%$ & $10 \%$ \\
\hline Impeded development & NEG & $8 \%$ & $5 \%$ & $10 \%$ \\
\hline Layoffs and reduction & NEG & $6 \%$ & $5 \%$ & $7 \%$ \\
\hline Declining well-being & NEG & $6 \%$ & $3 \%$ & $10 \%$ \\
\hline Increased business & POS & $8 \%$ & $8 \%$ & $7 \%$ \\
\hline Improved digitalization & POS & $5 \%$ & $3 \%$ & $7 \%$ \\
\hline Increased well-being & POS & $4 \%$ & $3 \%$ & $5 \%$ \\
\hline New insights & POS & $3 \%$ & $3 \%$ & $2 \%$ \\
\hline Remote working increased & MISC & $15 \%$ & $23 \%$ & $7 \%$ \\
\hline New work practices & MISC & $15 \%$ & $18 \%$ & $12 \%$ \\
\hline No big change & MISC & $6 \%$ & $3 \%$ & $10 \%$ \\
\hline
\end{tabular}

The theme New work practices show how organizations have been forced to find new solutions based on the pandemic. The answer "We had to plan new business concepts" shows signs of profoundly affected organizations. Answers also showed a perceived impact of New insights in the organization. One answer, for example, expressed a "Demand for truly agile consultancy raising".

\subsection{How Well Agility Helps to Respond to the Situation (Q3)}

Considering the perceptions of how well agility helps to respond to the pandemic situation (scale [not much, very well]), the majority $(55 \%(56 \%))$ of the responses $(n=134$ (89 of large/very large companies)) was on the supportive side (from the scale midpoint to "very well"), while $12 \%(13 \%)$ was from the scale midpoint to "not much". The distribution in large/very large companies was similar to the all. Notably, $16 \%(17 \%)$ reported that they do not know $(11 \%$ of the Finnish respondents $(\mathrm{n}=71)$ and $24 \%$ of the Swedish respondents $(\mathrm{n}=59))$.

\subsection{Further Insights}

Having presented the direct results of the survey, in this subsection, we take a deeper look at the different questions Q0-Q3 and analyze them in combination. This also informs further research (Sect. 5.4).

In order to investigate to which degree agility has helped organizations based on how challenging their impact of the pandemic was, we cross-analyzed the two questions Q1 and Q3, i.e., the relationship between the direction the pandemic impacted companies and how well agility has helped them to respond to the situation. It showed most respondents 
perceiving agility to have been helpful regardless of how (NEG or POS) the pandemic had an impact. To verify if there is a significant difference, we used the Kruskal-Wallis $\mathrm{H}$ test which is a non-parametric alternative to One Way ANOVA. However, the KruskalWallis $\mathrm{H}$ test does not show a statistically significant difference $(\mathrm{H}=13.112 \mathrm{df}=10$, Asymp. Sig. $=0.217$ ). This means that the agile way of working has been considered as helpful both in organizations where the impact of the pandemic has been negative as well as positive.

By cross-analyzing the two questions Q0 and Q3, i.e., how companies' overall agility has changed during the past year and how well agility has helped them to respond to the situation, we also investigate a possible relation. A majority of respondents perceived agility to be helpful during the pandemic, and a majority have improved their companies' overall agility. The Kruskal-Wallis $\mathrm{H}$ test confirmed a statistically significant difference $(\mathrm{H}=15.278, \mathrm{df}=4$, Asymp. Sig. $=0.004)$, which means that respondents perceiving agility to be helpful in this situation have also improved their agility during the last year.

To summarize the perceived impacts, although the respondents have expressed both positive and negative impacts, a majority of the answers show negative perceived impacts to the organization.

\section{Discussion and Conclusions}

We assess our study, derive recommendations, and conclude with further work plans.

\subsection{Related Works}

In comparison to our survey question Q3 (Sect. 4.4), a recent industrial study reported that mature agile business units have been more successful (in terms of customer satisfaction, employee engagement, operational performance) in coping with the impacts of the pandemic crisis than non-agile ones [7]. Following established agile practices and cross-functional ways of working, they were able to quickly reprioritize the business targets and continue. We found more negative impacts (53\%) due to the pandemic reported from our respondents than positive (33\%).

The majority of our respondents were in large or very large companies. In contrast, in a recent study, one software startup company faced many uncertainties and demands for adaptation when it was forced to quickly change the previous co-located agile ways of working to remote working at home [4]. The key challenges were maintaining the teams' productivity and ability to continue delivering satisfying customer value. Furthermore, the company paid attention to employee well-being. Well-being was also one of our resulting themes in Q2 (Sect. 4.3).

An immediate impact of the pandemic for almost every company has been increased remote work - even changing to full-time work at home due to governmental rulings. [1]. An increase in remote working was also one of our observations (Sect. 4.3).

Considering agility development (Q0, Sect. 4.1), a German management survey discovered that perceived agility of projects during the early stages of the pandemic remained high and, on average, slightly increased [5]. This concurs with our findings. 


\subsection{Recommendations}

Our study suggests that developing agility is useful both for and during turbulent times. It is Important to discern the type of change (internal or external) and its dynamic nature (factors increasing or decreasing, possibly even in both ways).

Business Agility is, by definition, an ability to sense and respond to changing business conditions quickly. Agility is also a mindset to react and move faster. This may be an explanation why the organizations that are agile and that have agile mindset have been coping better with the changing conditions due to the pandemic: the organization's structures and decision-making already support reacting quickly to changing conditions. The personnel is rather rewarded for taking action than punished for initiatives.

Furthermore, innovation is an inherent element of agile business and, under the pandemic circumstances, its role may have been amplified in companies to continue their businesses. However, such factors as extensive remote working and consequent co-operation difficulties discovered in this survey data (Sect. 4.3) may have had negative impacts on innovation performance.

\subsection{Limitations and Threats to Validity}

We did not ask directly about large-scale agile. However, $66 \%$ of our respondents were in large/very large companies and scaled agile methods are often used (Fig. 1).

We have recognized the limitations and potential threats earlier [8]. Especially, a limitation is that we did not ask the respondents to identify their organizations. Therefore, we cannot tell the number of different organizations in our respondent population, and we refrain from judging how representative our industrial sample is. Due to such statistical validity limitations, we make no attempt at generalizing the findings.

A construct validity concern is whether all the respondents in different roles and companies have interpreted all the terms in our questionnaire in the same way (e.g., 'agility'). We do not consider internal validity to be a significant concern since the purpose of the survey is primarily exploratory rather than explanatory. We have thus been cautious not draw decisive conclusions in this study. External validity judgement is limited by the background information collected. Research comparisons with other industrial surveys should consider possible biases. Due to the company-specific call-out of the survey (see Sect. 3), sampling bias is a threat. With the social media distribution, the response rate is unspecified. Considering reliability, the main concerns in this survey are thus the formulation of the question items and the dissemination.

\subsection{Further Research}

The mindset and culture of the agile organization is a possible area for further research based on our survey data. Related to that, one of our survey questions was: To what extent is Your company culture supporting agile ways of working, methods and practices? This question could give insights into whether a supporting company culture for agility has helped in managing the pandemic.

Another area for future research, based on our survey data, is to investigate answers to the survey question: Is the company as agile as it should be? Cross-analyzing this 
question with the perceived impacts (Sect. 4.2) could bring further understanding to whether the agile maturity has had an impact on managing the pandemic (Sect. 4.4).

Truly agile companies may have been more successful in continuous innovation. In this questionnaire we had the following multi-choice question related to that: What goals does the company attempt to achieve by agile means? One predefined answer choice was: New business (product and service innovation). There may be new business opportunities following the recovery of the pandemic to a "new normal".

\section{References}

1. Ralph, P., et al.: Pandemic programming: how COVID-19 affects software developers and how their organizations can help. Empir. Softw. Eng. 25(6), 4927-4961 (2020)

2. Bao, L., Li, T., Xia, X., Zhu, K., Li, H., Yang, X.: How does Working from Home Affect Developer Productivity? A Case Study of Baidu During COVID-19 Pandemic. arXiv preprint arXiv:2005.13167v2 (2020)

3. Forsgren, N.: Octoverse spotlight: an analysis of developer productivity, work cadence, and collaboration in the early days of covid-19. https://github.blog/2020-05-06-octoverse-spo tlight-an-analysis-of-developer-productivity-work-cadence-and-collaboration-in-the-earlydays-of-covid-19/. Accessed 9 March 2021

4. da Camara, R., Marinho, M., Sampaio, S., Cadete, S.: How do Agile Software Startups deal with uncertainties by Covid-19 pandemic? Int. J. Softw. Eng. Applications (IJSEA) 11(4), 15-34 (2020)

5. Schmidtner, M., Doering, C., Timinger, H.: Agile working during COVID-19 pandemic. IEEE Eng. Manag. Rev. https://doi.org/10.1109/EMR.2021.3069940

6. Worley, C.G., Jules, C.: COVID-19's uncomfortable revelations about agile and sustainable organizations in a VUCA world. J. Appl. Behav. Sci. 56(3), 279-283 (2020)

7. Handscomb, C., et al.: An operating model for the next normal: lessons from agile organizations in the crisis. https://www.mckinsey.com/business-functions/organization/our-ins ights/an-operating-model-for-the-next-normal-lessons-from-agile-organizations-in-the-crisis. Accessed 13 Feb 2021

8. Kettunen, P., Laanti, M., Fagerholm, F., Mikkonen, T., Männistö, T.: Industrial agile transformations lacking business emphasis: results from a Nordic survey study. In: Klotins, E., Wnuk, K. (eds.) ICSOB 2020. LNBIP, vol. 407, pp. 46-54. Springer, Cham (2021). https://doi.org/ 10.1007/978-3-030-67292-8_4

Open Access This chapter is licensed under the terms of the Creative Commons Attribution 4.0 International License (http://creativecommons.org/licenses/by/4.0/), which permits use, sharing, adaptation, distribution and reproduction in any medium or format, as long as you give appropriate credit to the original author(s) and the source, provide a link to the Creative Commons license and indicate if changes were made.

The images or other third party material in this chapter are included in the chapter's Creative Commons license, unless indicated otherwise in a credit line to the material. If material is not included in the chapter's Creative Commons license and your intended use is not permitted by statutory regulation or exceeds the permitted use, you will need to obtain permission directly from the copyright holder.

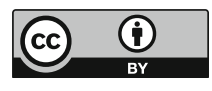

